\title{
The Phase Induced Amplitude Apodization Coronagraph: an overview of simulations and laboratory effort.
}

\author{
Eugene A. Pluzhnik ${ }^{1,6}$, Olivier Guyon ${ }^{1}$, Stephen Ridgway ${ }^{2}$, \\ Robert Woodruff ${ }^{3}$, Celia Blain ${ }^{1}$, Frantz Martinache ${ }^{4}$, \\ and Raphael Galicher ${ }^{5}$ \\ ${ }^{1}$ Subaru Telescope, National Astronomical Observatory of Japan, 650 North A'ohoku Place, \\ Hilo, HI 96720, USA, email: pea@naoj.org \\ ${ }^{2}$ National Optical Astronomical Observatories \\ ${ }^{3}$ Lockheed Martin Space Corporation \\ ${ }^{4}$ Observatoire de Haute Provence \\ ${ }^{5}$ Ecole Normale Supérieure, Paris, France \\ ${ }^{6}$ Institute of Astronomy of Kharkov National University, Sumskaya 35, Kharkov 61022,
} Ukraine

\begin{abstract}
The high contrast (typically $10^{10}$ ) and small angular separation between a planet and its parent star are the main challenges that need to be overcome to detect and characterize Earth-like planets around the nearest stars. Therefore, exoplanet imaging requires the use of a coronagraph, that ideally efficiently cancels the light from the star and has minimal influence on the planet image. The Phase Induced Amplitude Apodization Coronagraph relies on pupil apodization by geometrical remapping of the flux in the pupil plane. This method combines the advantages of classical pupil apodization with high throughput $(\approx 100 \%)$ and high angular resolution $(\approx \lambda / D)$, and has some unique advantages over most coronagraphs, such as low chromaticity, low sensitivity to stellar angular size and to small pointing errors. As a result, planet detection time is about 50-100 times shorter in comparison with classical coronagraphic techniques (Martinache et al. 2005).

Both the advantages of the PIAAC and the main factors affecting the performance of the coronograph will be examined in our laboratory experiment in which high quality PIAA optics wilkl be combined with wavefront control to demonstrate achromatic high contrast imaging $\left(10^{6}\right.$ or more) at small angular separation (less than $\left.2 \lambda / D\right)$. We present here a description and current status of this experiment together with a short analyses of the main factors affecting the performance of the coronograph.
\end{abstract}

Keywords. direct exoplanet imaging, coronagraphy, apodization, pupil remapping, diffraction propagation.

\section{Introduction}

The main goal of the Terrestrial Planet Finder mission (NASA) is to find and characterize Earthlike planets around the nearest stars. However detection of such planets is difficult because of the high contrast (typically $10^{10}$ ) and small angular separation (typically 50 mas) between the planet and its parent star. Therefore exoplanet imaging requires the use of a coronographic device. The Phase Induced Amplitude Apodization Coronagraph (PIAAC)(Guyon 2003) which relies on a loseless apodization technique, seems especially adapted to this task. Preliminary numerical and laboratory experiments with diamond turned lenses have confirmed the basic concept which this approach is based on (Galicher et al. 2005). However it was impossible to obtain high contrast with low-quality lenses. In the next phase of our laboratory experiment effort, high quality 


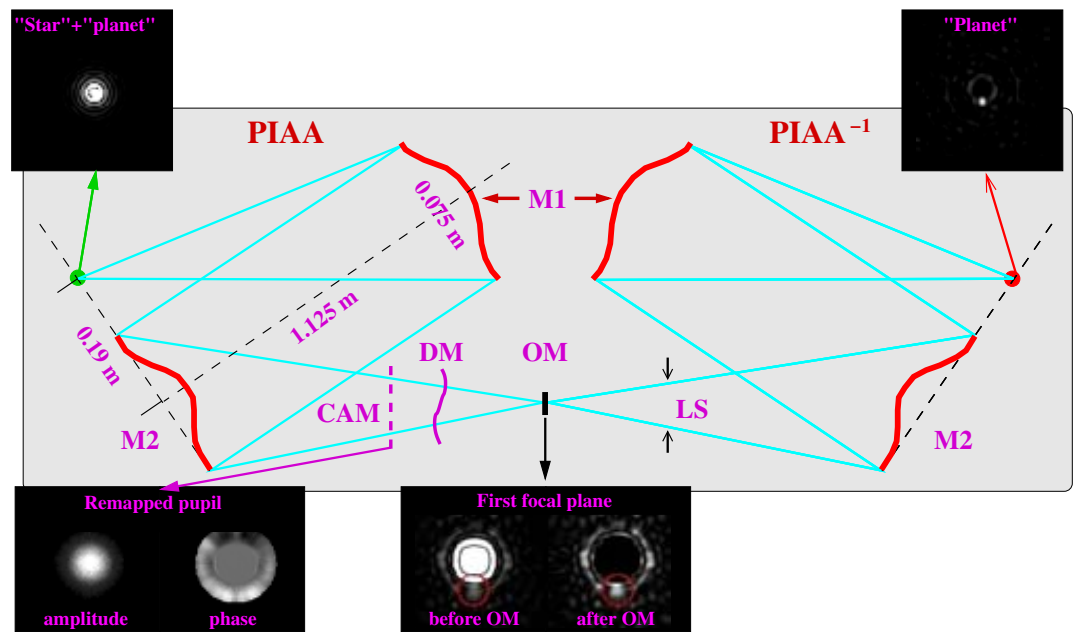

Figure 1. The optical layout of the PIAAC prototype. The maximal phase difference in the apodized pupil is 4.2 . The "planet" brightness is $10^{-4}$. The "star-planet" distance is $2 \lambda / D$.

mirrors will be combined with wavefront control to demonstrate achromatic high contrast imaging $\left(10^{6}\right.$ or more) at small angular separation (less than $\left.2 \lambda / D\right)$.

The goals of the experiment are to demonstrate/explore: (1) ability to polish the pupil remapping mirrors; (2) ability to correct off-axis abberations of the PIAAC with the second pupil remapping unit; (3) throughput, inner working angle and tip-tilt sensitivity of the PIAAC; (4) sensitivity to wavefront errors; (5) diffraction effects; (6) wavefront control with the remapped beam; (7) combination of the PIAAC with classical apodisation.

We present here a description and current status of our experiment together with analyses of the main factors affecting the performance of the coronograph, including diffraction effects, sensitivity to wavefront and pointing errors.

\section{Optical layout}

The experiment is designed to be operated in 3 modes: (1) without classical apodizer and Lyot mask (pure PIAA design); (2) without classical apodizer with Lyot mask (PIAA/Lyot design); (3) with classical apodizer and Lyot mask (PIAA/CPA design). The optical layout includes two identical Phase-Induced Amplitude Apodizers PIAA and PIAA $^{-1}$, (Fig. 1, Guyon et al. 2005), an occulting mask (OM) and a Lyot stop diaphragh (LS). The first PIAA apodizer, the occulting mask and the Lyot stop diaphragm produce a coronagraphic effect, while the second apodizer $\left(\mathrm{PIAA}^{-1}\right)$ corrects aberrations of offaxis sources introduced by the first apodizer. The source is collimated by the first PIAA mirror M1 and reimaged by the second PIAA mirror M2. The shape of the mirrors is a base off-axis paraboloid of revolution modified precisely to produce the designed output intensity profile. Each paraboloid has $1133 \mathrm{~mm}$ focal length and $75 \mathrm{~mm}$ diameter minumum clear aperture. The distance between the mirrors is $1125 \mathrm{~mm}$ and the centerline of the clear aperture is $190 \mathrm{~mm}$ off-axis. A deformable mirror (DM) is used to correct alignment and polishing errors. Additional apodization is obtained with the classical apodization mask CAM. Results of 3D raytracing simulations for amplitude /intensity and phase distributions in the main planes of the PIAAC are also presented in Fig. 1.

\section{Apodized beam profile}

Our goal is to achive a PSF contrast of $10^{6}$ at $2 \lambda / D$. Unfortunately, adopting a beam profile which exceed this requirement is dangerous because it increases the difficulty of 


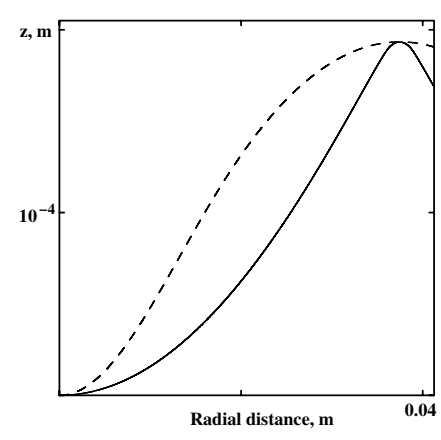

a)

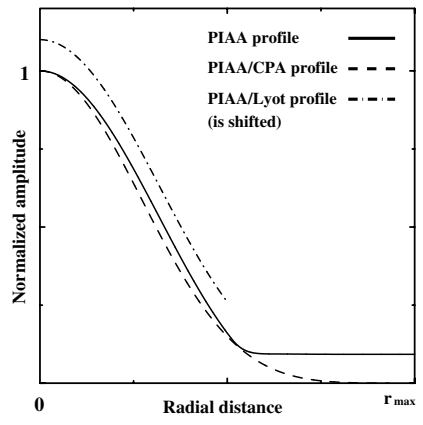

b)

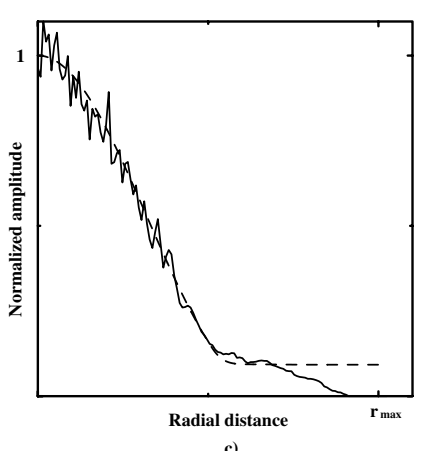

c)

Figure 2. The M1 (solid) and M2 (dashed) profile terms (a), designed amplitude profiles (b) and a simulated amplitude profile $(c)$ of the output beam.

polishing the optics. The most serious difficulty for the polishing of the optics is the sharp "bend" at the outside radius of the first mirror. This bend has a small radius of curvature and is therefore very challenging to polish, but it is essential because it distributes a small fraction of the entrance pupil's light into the broad wings of the output pupil.

In order to make the polishing of the optics easier we have chosen a hybrid design combining PIAAC and classical apodization. The classical apodization mask together with active wavefront control also can be used efficiently to correct the amplitude and phase errors produced with the first PIAA apodizer. This hybrid solution combines the advantages of both the PIAA and CPA, namely:(1) optics is easy to polish; (2) no significant loss in throughput; (3) no significant loss in angular resolution;

The chosen output beam profiles are presented in Fig. 2. The central part of the profile is a prolate function, which can be directly used in the PIAA/Lyot configuration, with maximal contrast of $10^{-6}$ at $2 \lambda / D$. PIAA/CPA mode allows imaging with contrast up to $10^{-8}$ at $2 \lambda / D$ (limited only by the wavefront errors).

\section{Mirror shapes}

The shape of both PIAA mirrors can be described by the equation:

$$
Z_{i}=\text { Off-axis_paraboloid }_{i}(x, y)+\text { Radial_profile }_{i}(r)+\text { Correcting_term }_{i}(x, y)
$$

where Off-axis_paraboloid ${ }_{i}(x, y)$ is the base shape of i-th mirror, Radial_profile ${ }_{i}(r)$ describes the modification of the base shape for on-axis system and Correcting_term $m_{i}(x, y)$ is the corrective term for an off-axis system.

For any desired apodisation profile the remapping function $r_{2}=f\left(r_{1}\right)$ can be determined such that the total flux within the radius $r_{1}$ of the input beam is equal to the total flux within the radius $f\left(r_{1}\right)$ of the output beam. This mapping provides us with a "correspondance table" between the radii at which geometrical light rays reflect on M1 and M2 mirrors for an on-axis source. For a given remapping function the radial profile terms are obtained by solving a differential equation (Guyon 2003)

$$
\frac{d M_{1}}{d r_{1}}=\frac{d M_{2}}{d r_{2}}=\sqrt{1+\frac{\left(M_{2}-M_{1}\right)^{2}}{\left(r_{2}-r_{1}\right)^{2}}}-\frac{M_{2}-M_{1}}{r_{2}-r_{1}}
$$

Corrective terms can be calculated by using an iterative algorithm. Exact radial profiles are presented in Fig. 2 together with simulated apodization profile for designed shapes. Note that the size of the first mirror is $10 \%$ larger size than maximal size of the beam. It gives us some flexibility to decrease diffraction propagation effects and simplify an analytical presentation of radial profiles used for diamond turning of the mirrors. 


\begin{tabular}{|c|c|c|}
\hline $\begin{array}{c}\text { Cycles per } \\
\text { aperture }\end{array}$ & $\begin{array}{c}\text { Maximum M2 surface } \\
\text { RMS error (contrast 10 }\end{array}$ & $\begin{array}{c}\text { Maximum output wavefront } \\
\text { RMS error (contrast 10-6) }\end{array}$ \\
\hline $0-5$ & $500 \mathrm{~nm}$ & $100 \mathrm{~nm}$ \\
$5-10$ & $300 \mathrm{~nm}$ & $100 \mathrm{~nm}$ \\
$10-20$ & $200 \mathrm{~nm}$ & $50 \mathrm{~nm}$ \\
$20-40$ & $70 \mathrm{~nm}$ & $35 \mathrm{~nm}$ \\
$>40$ & $50 \mathrm{~nm}$ & $25 \mathrm{~nm}$ \\
\hline
\end{tabular}

Table 1. The M2 surface quality and the output beam wavefront quality required to reach the $10^{-} 7$ and $10^{-} 6$ contrast respectively at $2 \lambda / D(\lambda=600 \mathrm{~nm})$ with the use of wavefront control.

\begin{tabular}{|l|cc|c|c|}
\hline & \multicolumn{2}{|c|}{$\begin{array}{c}\text { Shift }( \pm \mu \mathrm{m}) \\
(\mathrm{x}, \mathrm{y})\end{array}$} & $\mathrm{z}$ & Rotation \\
$( \pm \mu \mathrm{rad})$ & $\begin{array}{c}\text { Inclination } \\
( \pm \mu \mathrm{rad})\end{array}$ \\
\hline M1 & 5 & 47 & 41 & 3.2 \\
M2 & 14 & 60 & 52 & 4.5 \\
\hline
\end{tabular}

Table 2. Maximal alignment errors for the PIAAC prototype.

\section{Wavefront errors and alignment tolerance}

Errors in the surface of the mirrors produce both amplitude and phase errors in the output beam. It was found through numerical simulations that for the goal of our experiment amplitude errors in the output beam are negligible in comparison with phase errors. The RMS phase error in the output beam compatible with the $10^{-6}$ contrast at $2 \lambda / D$ is about $10^{-3} \lambda$ while a similar contrast degradation by amplitude errors would require 100 to 1000 times larger mirror surface errors. In Tables 1 we show phase errors tolerance for our experiment. Residual phase errors as well as alignment errors are corrected with the DM. In the aligned system the wavefront errors should not exeed 0.5 micron to be correctible with the DM. In Table 2 we show the alignment tolerance based on our raytracing simulations for $0.5 \mu \mathrm{m}$ wavefront errors. It has been identified through numerical simulations that these alignment errors can be easily corrected with the DM $(32 \times 32$ elements $)$ placed in the exit pupil. Amplitude errors can also be also corrected simultaneously with phase errors by the DM for half of the field of view. Our planed wavefront control scheme relies on focal plane wavefront sensing. The same focal plane array will be used for imaging and wavefront sensing. This approach provides optimal sensitivity for phase and amplitude sensing and offers optimal sensitivity to photon noise. We plan to use this technique to clear a "planet" search area out of speckles (details are given in (Guyon 2005)).

\section{Diffraction effects}

PIAA units have being designed and studied using geometrical optics approximations. However, differences between diffraction and geometrical propagation are not negligible at the $10^{-10}$ contrast level (Vanderbei 2005). Diffraction effects are introduced mainly by discontinuities or sharp transitions in the M1 mirror shape and can be easily observed on the M2 mirror surface. Diffraction is a source both amplitude and phase errors in output beam and can decrease coronagraphic performance. Since these diffraction effects are chromatic, they cannot be simultaneously corrected with the DM over a wide bandwidth.

There are two features of the output beam which can affect the high contrast PSF of an on-axis source (Fig. 3). The first one is high frequency oscillations at the edges of the output beam. The second is the peak/hole in the output beam center. It should be noted that these diffraction oscillations are mainly "sharp edges" effect. They decreases as the distanse from the edges increases and are smaller if boundaries are "smoother".

There are some possibilities to decrease diffractive oscillations (Pluzhnik et al. 2005):

(a) To use an oversized first mirror with an appropriate boundary profile (Fig. 4). 

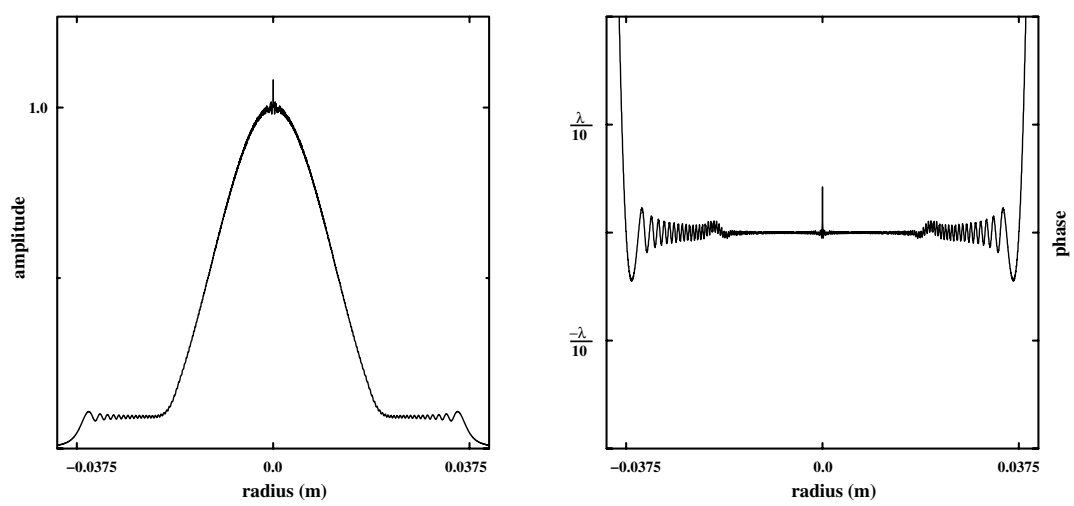

Figure 3. Diffraction effects in the PIAAC. The M1 mirror size is equal to the output beam size.
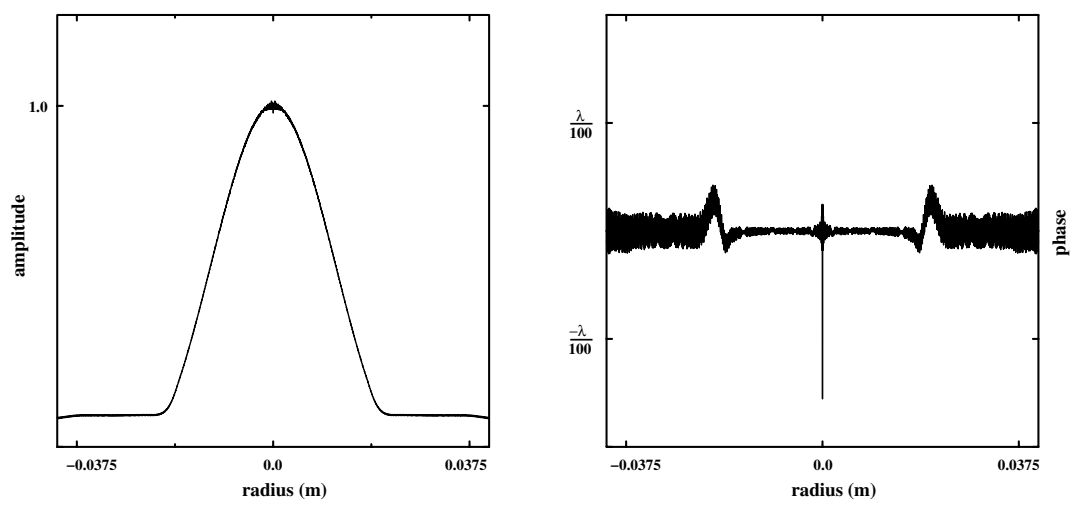

Figure 4. Diffraction effects for the PIAAC design with the "oversized" first mirror. The M1 mirror size is $10 \%$ larger than the output beam size.
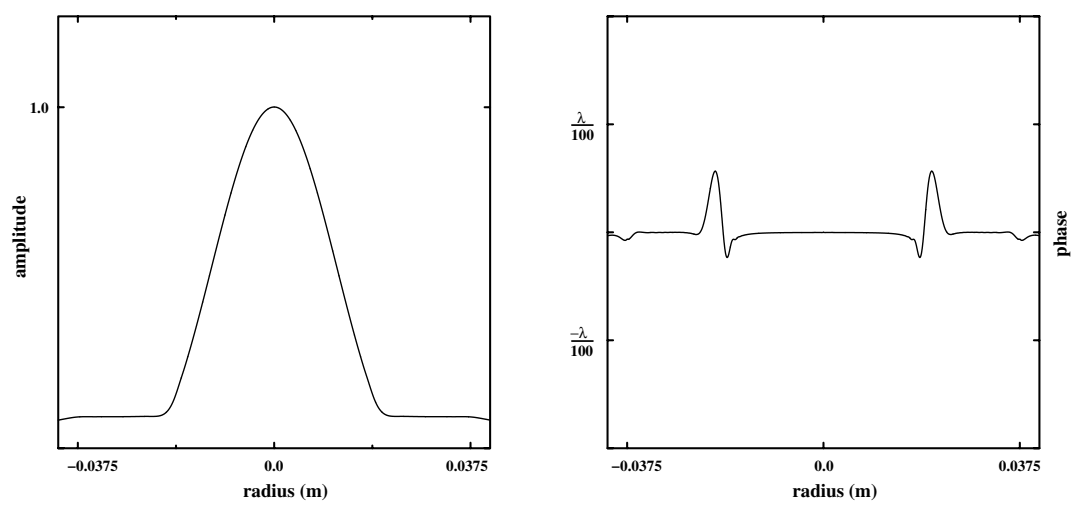

Figure 5. Diffraction effects for the "smoothed edge" PIAAC design. The sharp edge of the beam after the "oversized" M1 mirror is "smoothed" by using the $10 \%$ cosine taper window. The M1 mirror size is $10 \%$ larger than the output beam size.

(b) To smooth the first "oversized" mirror edge by using a classical apodization mask (Fig. 5).

It can be shown that it is possible to decrease diffraction effects to a level consistent with high-contrast $\left(10^{-10}\right)$ imaging by using an appropriate optical design of the PIAA 
unit. Such a design keeps all advantages of the PIAA coronagraph (Pluzhnik et al. 2005): high throughput (80-90\%, depending on the bandwith), small inner working angle (less then $2 \lambda / D)$, low chromaticity and relative insensitivity to pointing errors.

\section{Current status}

PIAA optics. Two set of PIAA mirrors are now beeing manufactured by AXSYS Technology, Inc. All mirrors have been diamond turned and verified by direct measurements of the surface profile. The overall shapes are in good agreement with our design with only $1 \lambda$ RMS of deviation. There is no excess of error in any area of the mirrors: all surfaces were accurately produced by diamond turning. The beam apodization after this mirror is in good agreement with our numerical simulations. The optics are under fine polishing right now and will be finished before the end of 2005 .

Light source. At the first stage of the experiment we are planing to use single point source, which will simulate an unresolved star. The $2 \lambda / d$ for our optics set is approximately equal to 19 microns, so the source can be easily made using a $2 \mu \mathrm{m}$ pinhole and a laser or a single mode fiber.

Classical apodization mask. A classical apodization mask is used to convert the "moderately" apodized beam into a fully apodized beam and, possibly, to correct slight errors of PIAA apodization. The mask only affects the outer parts of beam, where there is little light. As a result the overall transmition of the mask is high and it does not reduce the throughput and resolution of the system. The mask can be either continuous or binary, with no significiant difference of performance between them. Another mask (preapodizer) will be used to decrease diffraction effects in the PIAA optics.

Deformable mirror. The 1024 elements MEMS DM manufactured by Boston Micromachines will be used to correct alignment errors and cancel speckles in the "planet" search area. Electronics and control software for the DM control is developed with support of the Subaru telescope Adaptive Optics group.

\section{Summary}

The current status of the PIAA optics laboratory experiment under preparation at Subaru Telescope has been reviewed in this paper. We have given a short analyses of the main factors affecting the performance of the coronograph as well as some ideas how to decrease their influence.

\section{Acknowledgements}

This work was carried out under JPL contract numbers 1254445 and 1257767 for Development of Technologies for the Terrestrial Planet Finder Mission, with the support and hospitality of the National Astronomical Observatory of Japan.

\section{References}

Galicher, R., Guyon, O., Otsubo, M., Suto, H., \& Ridgway, S. 2005, PASP, 117, 411

Guyon, O. 2003, A\&A, 404, 379

Guyon, O., Pluzhnik, E.A., Galicher, R., Martinache, F., Ridgway, S., \& Woodruff, R. 2005, ApJ, 622, 744

Guyon, O. 2005, ApJ, 629, 592

Martinache, F., Guyon, O., Pluzhnik, E.A., \& Galicher, R. Accepted by ApJ

Pluzhnik, E.A., Guyon, O., Ridgway, S., Martinache, F., Woodruff, R., Blain, C., \& Galicher, R. In preparation

Vanderbei, R.J. 2005, astro-ph/0506550 